Study of Nonlinear Evolution of the Weibel-like Instability of Relativistic Electron Beams Relevant to Fast Ignition and Astrophysics

M. Wei

December 19, 2012 
This document was prepared as an account of work sponsored by an agency of the United States government. Neither the United States government nor Lawrence Livermore National Security, LLC, nor any of their employees makes any warranty, expressed or implied, or assumes any legal liability or responsibility for the accuracy, completeness, or usefulness of any information, apparatus, product, or process disclosed, or represents that its use would not infringe privately owned rights. Reference herein to any specific commercial product, process, or service by trade name, trademark, manufacturer, or otherwise does not necessarily constitute or imply its endorsement, recommendation, or favoring by the United States government or Lawrence Livermore National Security, LLC. The views and opinions of authors expressed herein do not necessarily state or reflect those of the United States government or Lawrence Livermore National Security, LLC, and shall not be used for advertising or product endorsement purposes.

This work performed under the auspices of the U.S. Department of Energy by Lawrence Livermore National Laboratory under Contract DE-AC52-07NA27344. 


\title{
Study of Nonlinear Evolution of the Weibel-like Instability of Relativistic Electron Beams Relevant to Fast Ignition and Astrophysics
}

\author{
Dr. Mingsheng Wei, General Atomics \\ August 29, 2012
}

\section{INTRODUCTION}

Relativistic laser matter interaction generates an intense fast electron beam with Megaampere (MA) to Giga-ampere current. Such beam can propagate into high-density plasmas as background plasma electrons can provide sufficient return current to maintain charge and current neutrality. However, such system is subject to Weibel-like filamentation instabilities that break out the beam into filaments and generate intense magnetic fields. This could result in anomalous stopping and large angular spread of the electron beams, leading to a lower energy coupling to the fuel in the fast ignition (FI) scheme of inertial confinement fusion, therefore, of significant interest to FI. Understanding the nonlinear growth of the Weibel B-fields and its structure is also important to astrophysics as it is generally accepted to be responsible for relativistic collisionless shocks and cosmic rays and "jitter" radiation in gamma ray burst.

The anticipated high energy and high intensity NIF ARC laser pulse (4.3 kJ, $5 \mathrm{ps})$ can produce fast electron beam with a mean energy of $\sim 3 \mathrm{MeV}$ and a total current of $\sim 100 \mathrm{MA}$. We have performed a series of hybrid particle-in-cell (PIC) simulations to study NIF-ARC laser produced fast electron beam transport and examine the dependence of the nonlinear evolution of the Weibel-like fields and its structure on the background plasma density and temperature as well as the beam divergence. Significant fraction of energy is found to transfer to the Weibel B-fields and there is strong dependence on the plasma density. Simulations have also been performed to assess the feasibility of probing the Weibel-like fields with high-energy (e.g., $10 \mathrm{MeV}$ ) protons.

\section{BACKGROUND}

The Weibel instability is a fundamental collective plasma process through which particle kinetic energy can be transferred to magnetic energy. This instability was first investigated by $\mathrm{E}$. Weibel in 1959 where he predicted spontaneously growing electromagnetic fields in plasmas due to an anisotropic velocity distribution of electrons [1]. The physical mechanism of the Weibel instability is illustrated in Fig. 1. If there are two counter propagating electron streams in the $\mathrm{Y}$ direction, a transverse magnetic field in the $\mathrm{Z}$ direction can spontaneously arise from a noise source as a result of a perturbation in the net current. The transverse magnetic field will bend the electron trajectory due to the $\mathrm{v} \times \mathrm{B}$ force and lead to the aggregation of electrons reinforcing the perturbation which causes

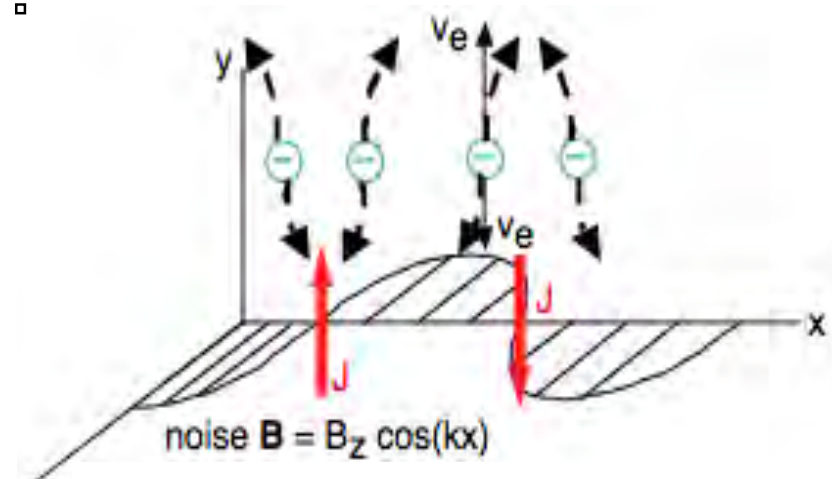

z Fig. 1 Physical mechanism of the Weibel. the further growth of the magnetic field, i.e., a positive feedback. The main consequence of this instability is to cause the electron beam to break-up into small filaments with a typical transverse dimension of the order of the collisionless skin depth, $c / \omega_{\mathrm{pe}}$ (where $\mathrm{c}$ is the speed of light and $\omega_{\mathrm{pe}}$ is the electron plasma frequency). 
The initial growth rate of the relativistic Weibel instability (WI) is given by

$$
\Gamma_{W I}=\frac{\beta_{b 0} \omega_{p 0}}{\sqrt{\gamma_{b 0}}} \sqrt{\frac{n_{b 0}}{n_{p 0}}}
$$

and can be much smaller than $\omega_{\mathrm{p} 0}$, where $\mathrm{n}_{\mathrm{b} 0}$ and $\gamma_{\mathrm{b} 0}$ are the initial beam density and relativistic factor, respectively; $\omega_{\mathrm{p} 0}$ and $\mathrm{n}_{\mathrm{p} 0}$ are the background plasma frequency and density. WI develops over a very short time scale, on the order of $1 / \omega_{\mathrm{p} 0}$ and the growth rate has a maximum at a wavelength $\sim \mathrm{c} / \omega_{\mathrm{p} 0}$ (the collisionless plasma skin depth) due to the kinetic effects. Many Particle In Cell (PIC) simulations have been performed to study the nonlinear evolution of the WI and the magnetic field structures in both fast ignition [2] and astrophysics scenario [3]. The electron beam are found to break into small-scale current filaments, and subsequently the filaments selforganize in a coaxial structure and are then guided by the quasi-static magnetic channels. These small filaments are subsequently observed to coalesce to form large-scale filaments.

In the context of ultra-high intensity short pulse laser solid interactions, both experiments and particle simulations have shown that the incident laser energy can be efficiently transferred to relativistic electrons with hundreds of $\mathrm{keV}$ to $100 \mathrm{MeV}$ kinetic energy. The high energy flux yields 10's - 100's MA beam currents. The beam density at the interaction region can be comparable to the critical density $\gamma \mathrm{n}_{\mathrm{c}}$ where $\gamma$ is the relativistic factor and $\mathrm{n}_{\mathrm{c}}=1.1 \times 10^{21} \mathrm{~cm}^{-3}$ for 1 $\mu \mathrm{m} \mathrm{Nd:glass} \mathrm{laser,} \mathrm{which} \mathrm{is} \mathrm{approximately} \mathrm{1/100th} \mathrm{of} \mathrm{solid} \mathrm{density.} \mathrm{The} \mathrm{incident} \mathrm{beam} \mathrm{intensity}$ can be 100 times higher than the Alfvén current limit $\left(\mathrm{I}_{\mathrm{A}}=17 \beta \gamma \mathrm{kA}\right)$. Consequently, a return current has to be provided by the dense target to maintain charge and current neutrality for the relativistic electron beam to propagate. Such counter-propagating streams are strongly subject to the Weibel instability as seen in numerous PIC simulations [3]. The WI could play an important role in the fast ignition scenario as it can result in filamentation and magnetic field generation that would lead to collective energy loss of a relativistic electron beam in both coronal and core plasma region. Electron filaments have been observed both directly and indirectly in high intensity laser matter interaction experiments [4]. However, so far, the experimental data is scarce due to the limited capabilities on plasma sample creation and lack of advanced diagnostics to detect the magnetic fields with high spatial and temporal resolution. Effects of electron beam and background plasma parameters on the evolution of WI have never been investigated systematically.

Weibel instability plays an important role in astrophysical plasmas. Many astrophysical objects such as gamma ray bursts, jets from active galactic nuclei, jets from quasars and supernova remnants emit non-thermal radiation, which is believed to be emitted in strong collisionless shocks. The observed non-thermal radiation indicates that strong particle acceleration and magnetic field generation in these shocks. Weibel instability has been suggested as a mechanism for relativistic collisionless shock formation and shock dynamics depends on the long-term evolution of the magnetic fields. Various analytical models and PIC simulations [3] have shown that the Weibel instability triggers collisionless shock formation in non-magnetized plasmas, formation of the electron filaments and merging of small filaments, and the generation of magnetic fields. The Weibel instability is also suggested as a key part of the dynamics in generating the relativistic shocks thought to be the source of energetic radiation emerging from gamma ray burst [3]. Weibel instability has also been proposed to be the trigger for "jitter radiation", which is suggested to emerge from gamma ray bursts.. 


\section{OBSERVATION}

\section{A. Simulations set up}

2D hybrid PIC simulations using the LSP code [5] are performed in Cartesian geometry with $1000 \mu \mathrm{m}$ in $\mathrm{X}$ (longitudinal direction) and $200 \mu \mathrm{m}$ in $\mathrm{Y}$ (transverse) with cell size as small as $0.25 \mu \mathrm{m}$. Background plasma is $\mathrm{CH}$ with various densities, i.e., $0.2 \mathrm{~g} / \mathrm{cc}, 0.6 \mathrm{~g} / \mathrm{cc}$ and $1.0 \mathrm{~g} / \mathrm{cc}$ and temperature, i.e., $20 \mathrm{eV}, 60 \mathrm{eV}$ and $100 \mathrm{eV}$. Fast electrons are treated kinetically while background plasmas are fluid.

ARC beam with the design parameters of $4.3 \mathrm{~kJ}$ in 5 ps focused to a $40 \mu \mathrm{m}$ spot with the interferometric adaptive optics systems [6] will achieve the nominal peak intensity of $6.8 \times 10^{19}$ $\mathrm{W} / \mathrm{cm}^{2}$. The expected relativistic factor is about 7 and the critical density is $7.7 \times 10^{21} \mathrm{~cm}^{-3}$ (also the number density of the fast electrons that will be generated by the $\mu \mathrm{m} \mathrm{ARC).} \mathrm{From} \mathrm{the}$ ponderomotive scaling law [7], relativistic electron beam produced from the interaction of the ARC beam with a thin solid foil target will have an average kinetic energy of $k T_{h o t}(M e V)=0.511 \times\left(\sqrt{1+\frac{I \lambda_{\mu m}^{2}}{1.37 \times 10^{18} \mathrm{Wcm}^{-2}}}-1\right) \approx 3 \mathrm{MeV}$. With an energy conversion efficiency of $\eta \sim 30 \%$, the total energy carried by the relativistic electron beam will be about $1.3 \mathrm{~kJ}$, which will result in a peak hot current density $\mathrm{J}_{\text {hot }}=\eta\left(\mathrm{I}_{\text {laser }}\right) / \mathrm{kT}_{\text {hot }} \approx 7.6 \times 10^{12} \mathrm{~A} / \mathrm{cm}^{-2}$ and a total current of about 95 MA. Such intense fast electron beam (5 ps flat temporal profile) is injected into the background plasmas. Two different divergence half angles, i.e., $30^{\circ}$ and $15^{\circ}$, for the fast electron beam are used to study the dependence of the field growth on the initial fast electron beam divergence.
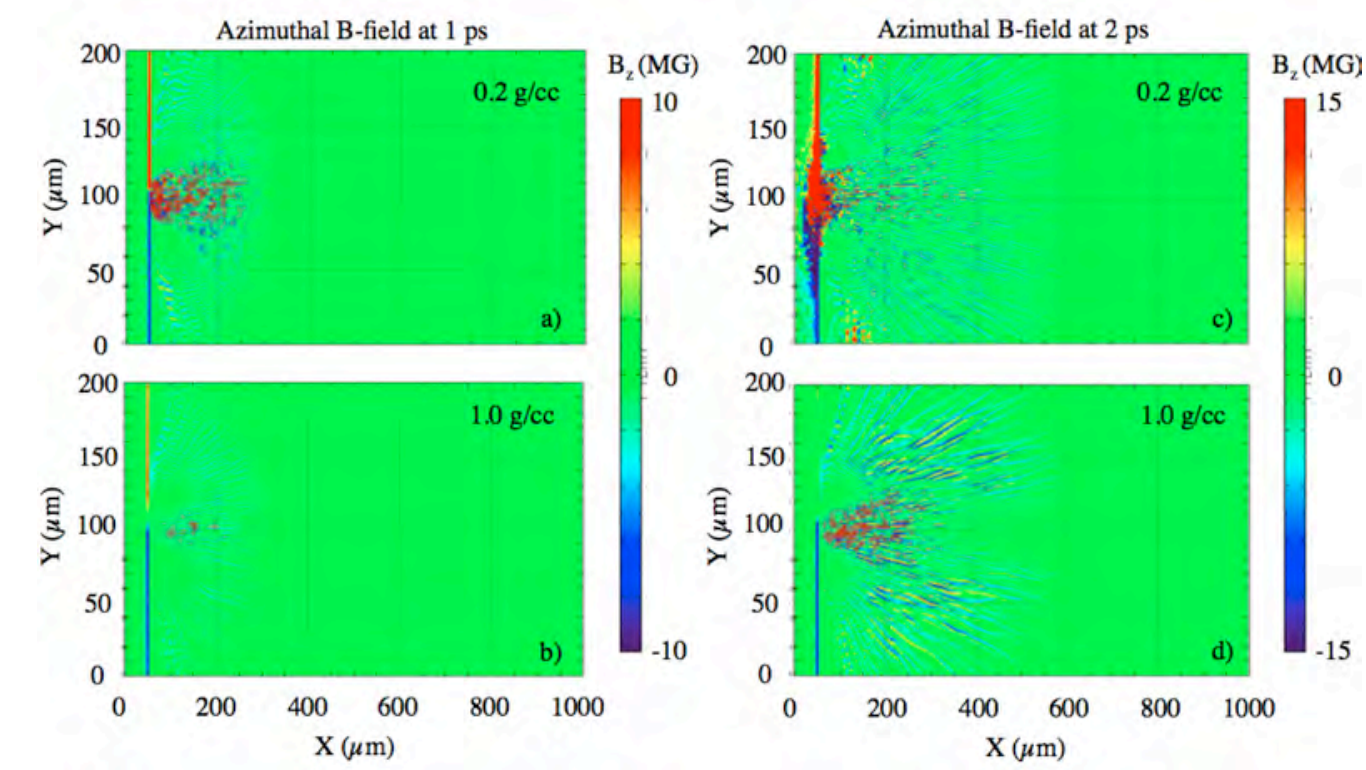

Fig. 2 Azimuthal B-field development as the result of the fast electron beam transport in $\mathrm{CH}$ plasmas with two different densities, i.e. $0.2 \mathrm{~g} / \mathrm{cc}$ and $1.0 \mathrm{~g} / \mathrm{cc}$ at 1 and $2 \mathrm{ps}$.

\section{B. Dependence on the background plasma density}


We observed very strong dependences of the Weibel field development with the background plasma density, particularly with the plasma density comparable to the beam density, which is consistent with the theory discussed in the Background section. Fig. 2 compares azimuthal magnetic fields at $1 \mathrm{ps}$ and $2 \mathrm{ps}$ for two different background plasma density cases, i.e., $0.2 \mathrm{~g} / \mathrm{cc}$ and $1.0 \mathrm{~g} / \mathrm{cc}$. For those simulations, fast electron beam divergence was kept at $30^{\circ}$ and the initial plasma temperature was $100 \mathrm{eV}$. We clearly see that fast electron beam instantly breaks out into many $\mu \mathrm{m}$-scale filaments inside the $\mathrm{CH}$ plasmas. Lower density $(0.2 \mathrm{~g} / \mathrm{cc})$ background plasma results in much stronger Bfields in both early time (1 ps) and a later time (2

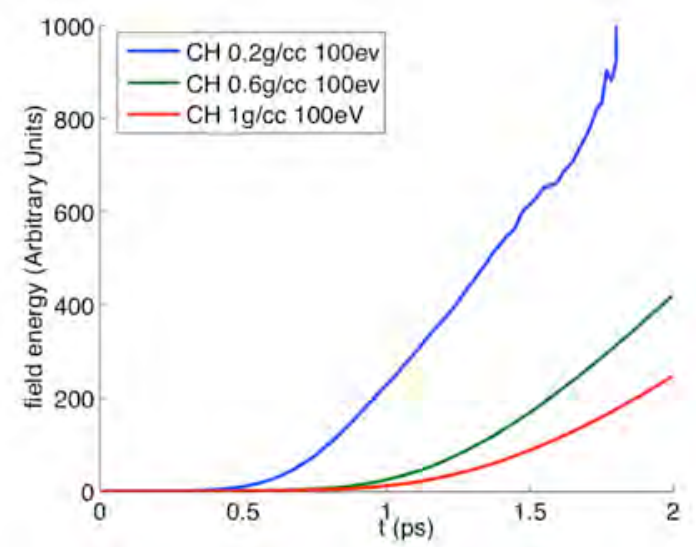

Fig. 3 Time evolution of the B-field energy in three different background plasma densitv cases.

ps) close to the region where the beam was injected. While in the $1 \mathrm{~g} / \mathrm{cc}$ density case, fast electrons can transport more efficiently further into the background plasmas. We see relatively stronger Weibel-like B-fields developed later time (e.g. 2 ps) inside the solid density $\mathrm{CH}$ plasmas associated with fast electron beam transport. Time evolution of the B-field energy shown in Fig. 3 clearly demonstrate a much fast growth of the WI fields and a factor of 4 increase in field energy at the $0.2 \mathrm{~g} / \mathrm{cc}$ case compared to that in the $1.0 \mathrm{~g} / \mathrm{cc}$ background plasma density case. Fast electron beam transport in the lower background density case is severely inhibited as shown in Fig. 4.

\section{Effect of the background plasma temperature}

Simulations were performed with the same background plasmas density (1 $\mathrm{g} / \mathrm{cc}$ ) but with various initial plasma temperatures, i.e., $20 \mathrm{eV}, 60 \mathrm{eV}$ and 100

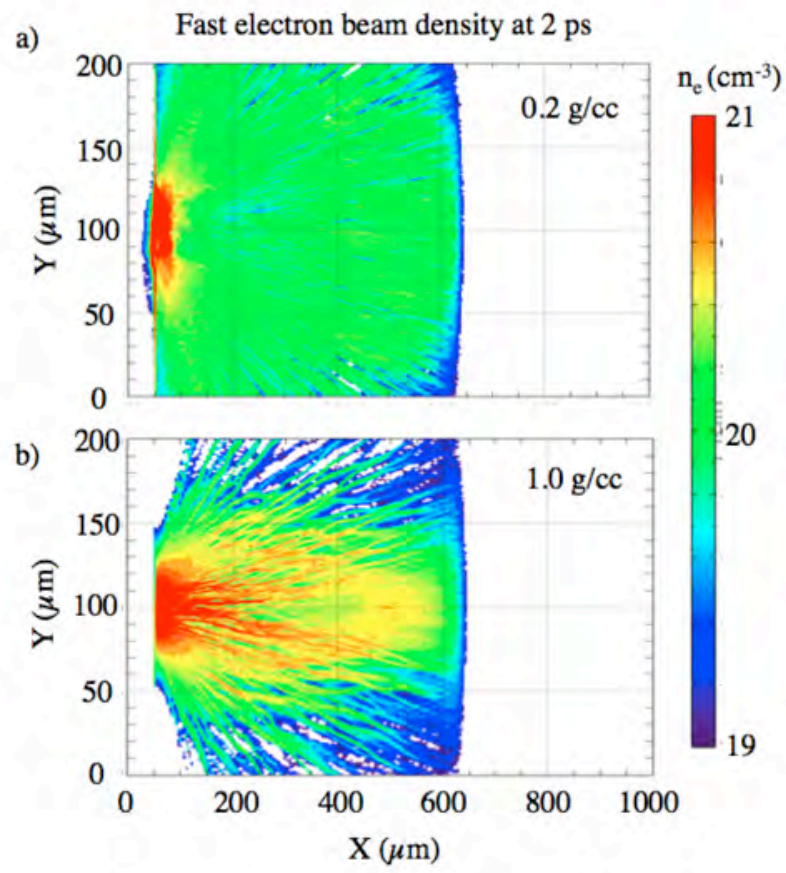

Fig. 4 Contour plots of the fast electron number density at $2 \mathrm{ps}$ in the $0.2 \mathrm{~g} / \mathrm{cc}$ and $1.0 \mathrm{~g} / \mathrm{cc}$ background plasma density cases.

$\mathrm{eV}$. No clear trend was observed as shown in the time history of the accumulated B-field energy (Fig. 5). This can be attributed to rapid resistive heating of background plasmas with an intense fast electron beam at high current density. 
口

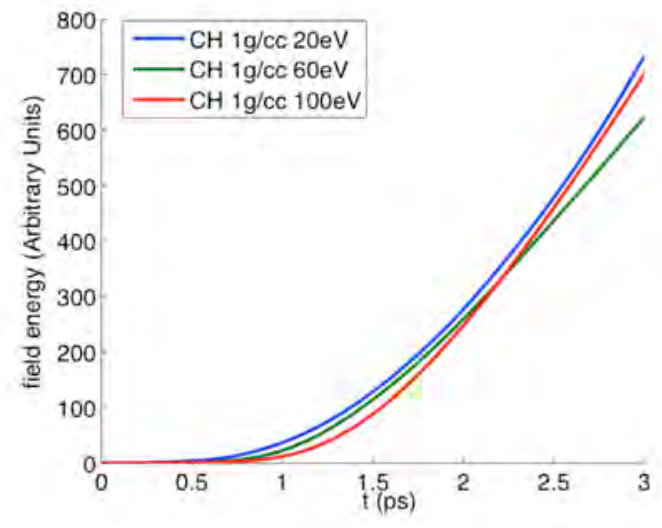

Fig. 5 Time evolution of the B-field energy in three different background plasma temperature cases.
口

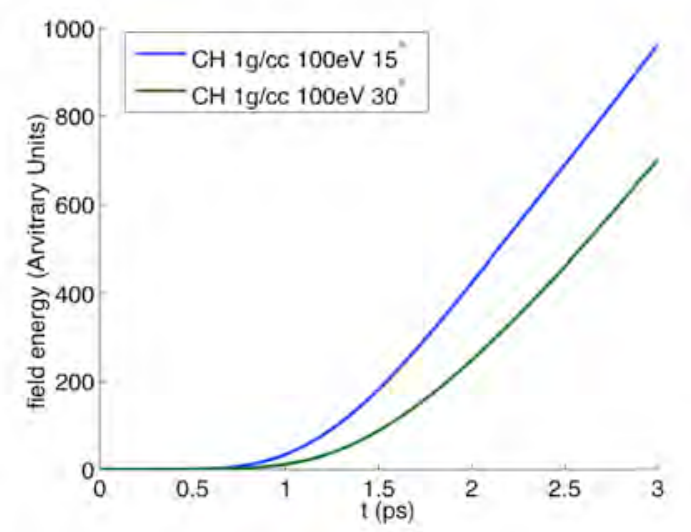

Fig. 6 Time evolution of the B-field energy in simulations with fast electron beam divergence of $15^{\circ}$ and $30^{\circ}$.

\section{Effect of the fast electron beam divergence on the Weibel-like field development}

Simulation was also performed with a smaller beam divergence $\left(15^{\circ}\right)$ to compare results from a standard calculation with a $30^{\circ}$ divergence angle. The background $\mathrm{CH}$ plasma density and the initial temperature were $1 \mathrm{~g} / \mathrm{cc}$ and $100 \mathrm{eV}$ respectively. We found that the propagation of fast electron beam in solid density plasmas with a small beam divergence can induce more intense B-fields (due to a large gradient in fast electron current density) as shown in the time history of the B-field energy plot (Fig. 6) and the azimuthal B-fields contour plot (Fig. 7). Electron beam in this case is guided by the intense self-generated B-fields that lead to a more efficient energy transport as shown in the fast electron number density contour plot in Fig. 7(d). 口
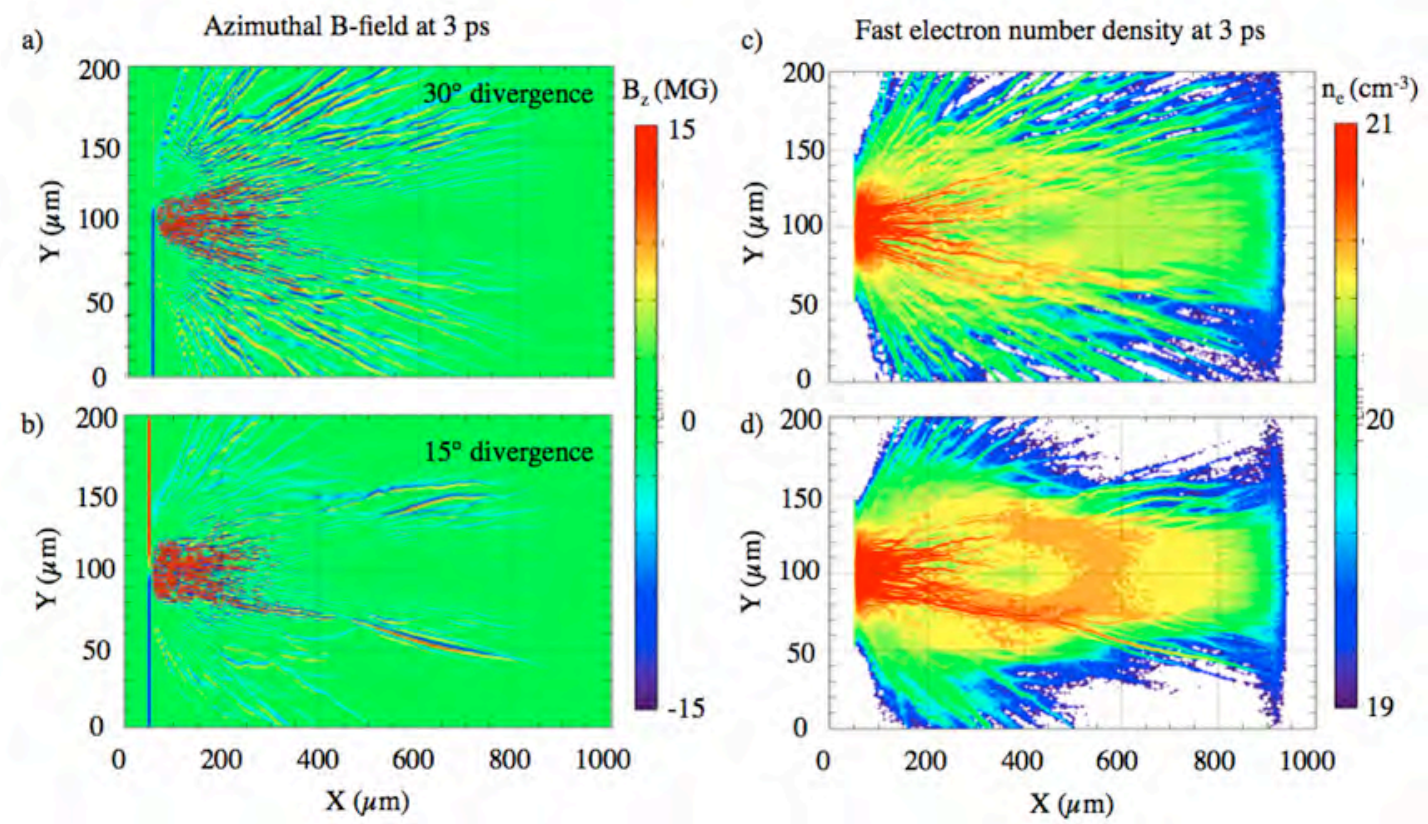

Fig. 7 Comparison of azimuthal B-fields (a) and (b) and fast electron density (c) and (d) at $3 \mathrm{ps}$ in simulations with fast electron beam divergence at $30^{\circ}$ and $15^{\circ}$ respectively. 
Fast electrons are also found to accumulate on-axis which can be attributed to B-field deflecting electrons toward the center.

口
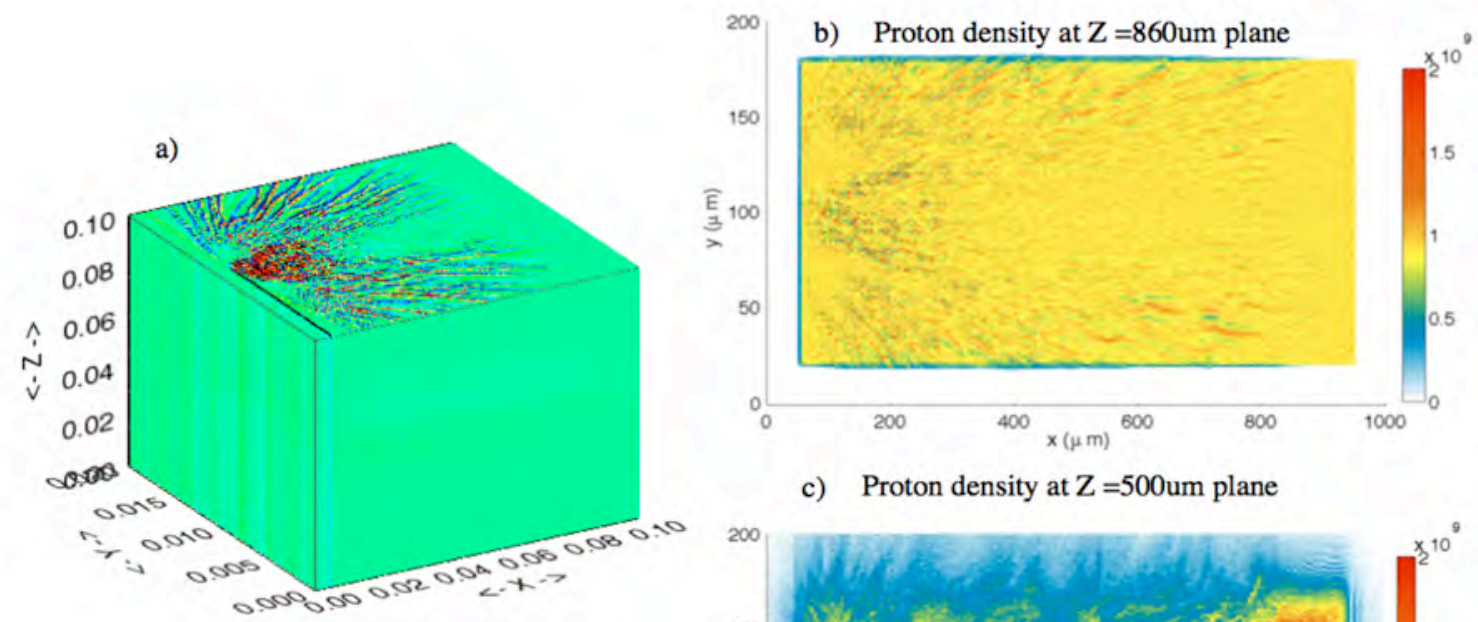

c) Proton density at $\mathrm{Z}=500 \mathrm{um}$ plane

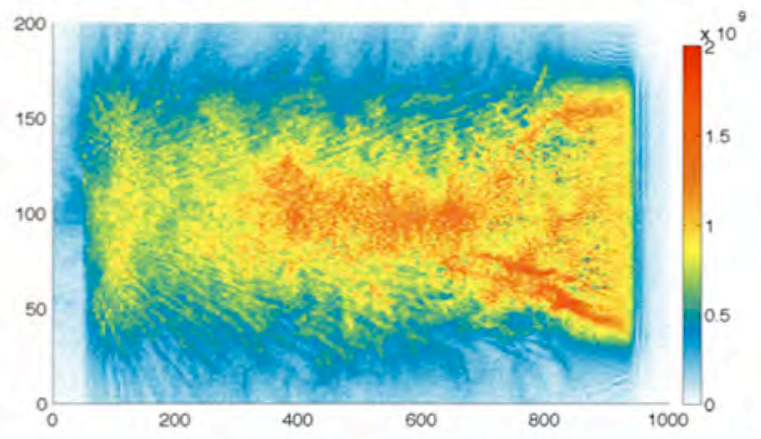

Fig. 8 (a) $\mathrm{B}_{\mathrm{y}}$ fields stacked along $\mathrm{Z}$ direction in 3D LSP simulations and the simulated proton bema density (time accumulated) distribution at $\mathrm{Z}=860 \mu \mathrm{m}$ plane (b) and at $\mathrm{Z}=500 \mu \mathrm{m}$ plane (c).

\section{E. Detect fast electron beam generated B-field and its structures with proton probe}

Particle probe using both the implosion produced mono-energetic protons [8] and the high intensity ps laser produced broader energy range protons [9] have been widely used to probe the implosion, laser produced plasmas and the associated electromagnetic fields. The data interpretation however is not straightforward due to the complex field structures. We have performed preliminary 3D LSP simulations to evaluate the effectiveness of proton probing technique for measuring the field strength and imaging the complex field structures observed in our transport modeling shown above. Simulations were in 3D Cartesian geometry as shown in Fig. 8(a). To facilitate simulations, 2D spatial profile of a typical azimuthal B-field (Fig. 7(a)) was stacked $\mathrm{Z}$ direction with B-field set along Y. Background plasmas were not modeled. A 10 $\mathrm{MeV}$ proton beam with a uniform density was injected downward, i.e., propagating in $-Z$ direction from $X Y$ plane at $Z=900$. Under this arrangement, protons will be deflected along $X$ direction. The time integrated proton beam density distribution on the $X Y$ planes along various $Z$ locations was examined. Fig. 8(b) shows the accumulated proton density distribution at $Z=860$ $\mu \mathrm{m}$ plane. The modulations seen in the proton beam density distribution agree well with the structures in the $\mathrm{B}_{\mathrm{y}}$ fields. At later time, protons were found to accumulate in regions where the B-fields were weak as shown in Fig. 8(c).

The displacement distance along $\mathrm{X}$ direction obtained from the proton image at various Z-planes can be used to estimate the average B-fields. For example, we observe about $200 \mu \mathrm{m}$ 
deflection distance in the $\mathrm{X}$ direction at $\mathrm{Z}=500 \mu \mathrm{m}$ plane after a propagation distance of $400 \mu \mathrm{m}$. This requires $\sim 10 \mathrm{MG}$ fields, consistent with the field magnitude we had in the simulations.

It should be noted that deflection of protons in a real 3D experiment will be more complicated as the fields structures will be incoherent, unlike the simple case we used here. To fully interpret the proton probing data will require 3D simulations with truly 3D fields structures. Nonetheless, the technique shown here should be still valid.

\section{CONCLUSION}

We have performed a series of hybrid PIC simulations to study NIF-ARC laser produced fast electron beam transport and examine the dependence of the nonlinear evolution of the Weibel-like fields and its structure on the background plasma density and temperature as well as the beam divergence. Strong dependence on plasma density and fast electron beam divergence was observed. Low background plasma density leads to strong grow of the Weibel fields near the beam creation plane and significantly inhibit fast electron transport. Small fast electron beam divergence results in strong self-generated B-fields that can guide fast electron transport. We have also demonstrated the feasibility of probing the Weibel-like field structures with highenergy (e.g., $10 \mathrm{MeV}$ ) protons in 3D geometry. In summary, we have completed all the proposed work described in the Statement Of Work of this NIF Concept Exploration project.

\section{DISSEMINATION OF THE RESULTS}

The results will be presented at the international workshop and conference such as the 12th International Workshop on Fast Ignition of Fusion Targets, Nov. 4-8, 2012 in Napa Valley, CA. A publication in peer-reviewed journal is planned.

\section{ACKNOWLEDGEMENT}

This work was funded by DOE/NNSA under Contract No. DE-AC52-07NA27344 via a LLNL subcontract \#B598295.

\section{REFERENCES}

1. E. W. Weibel, Phys. Rev. Lett. 2, 83 (1959)

2. M. Tabak et al., Phys. Plasmas 1, 1626 (1994); B.F. Lasinski et al., Phys. Plasmas 6, 2041 (1999); Y. Sentoku et al., Phys. Plasmas 7, 689 (2000); M. Honda et al., Phys. Rev. Lett. 85, 2128 (2000); T. Taguchi et al., Phys. Rev. Lett. 86, 5055 (2001); C. Ren et al., Phys. Rev. Lett. 93, 185004 (2004); J.J. Honrubia and J. Meyer-ter-Vehn, Nucl. Fusion 46, L25 (2006); O. Polomarov et al., Phys. Plasmas 14, 043103 (2007); A.A. Solodov, R. Betti, Phys. Plasmas 15, 042707 (2008); G. Shvets et al., Phys. Plasmas 16, 056303 (2009)

3. M.V. Medvedev and A. Loeb, Astrophys. J. 526, 697 (1999); M.V. Medvedev et al., Astrophys. J. Lett. 618, L75 (2005); L.O. Silva et al., Phys. Plasmas 9, 2458 (2002); R. A. Fonseca et al., Phys. Plasmas 10, 1979 (2003); M. Milosavljevic et al., Astrophys. J. 637, 765 (2006); A. Spitovsky, Astrophys. J. Lett. 673, L39 (2008)

4. M.S. Wei et al., Phys. Rev. E 70, 056412 (2004); M. Tatarakis et al., Phys. Rev. Lett. 90, 175001 (2003); R. Jung et al., Phys. Rev. Lett. 94, 195001 (2005) 
5. D.R. Welch et al., Phys. Plasma 13, 063105 (2006); T. P. Hughes et al., Phys. Rev. ST Accel. Beams, 2, 110401 (1999); D. R. Welch et al., Nucl. Instrum. Methods Phys. Res. A, 464, 134 (2001)

6. K.L. Baker et al., Proc. of SPIE, 7209, 720907 (2009)

7. S.C. Wilks et al., Phys. Rev. Lett. 691383 (1997)

8. C.K. Li et al., Rev. Sci Instrum. 77, 10E725 (2006); C.K. Li et al., Phys. Rev. Lett. 97, 135003 (2006); C.K. Li et al., Phys. Rev. Lett. 99, 015001 (2007); C.K. Li et al., Phys. Rev. Lett. 100, 225001 (2008); C.K. Li et al., Phys. Plasmas 16, 056304 (2009); C.K. Li et al., Plasma Phys. Control. Fusion 51, 014003 (2009); Petrasso et al., Phys. Rev. Lett. 103, 085001 (2009)

9. M. Borghesi et al., Rev. Sci. Instrum. 74, 1688 (2003); A. J. Mackinnon et al., Rev. Sci. Instrum. 75, 3531 (2004); L. Romagnani et al., Phys. Rev. Lett. 95, 195001 (2005); M. Borghesi et al., Laser Part. Beams 25, 291 (2007); L. Romagnani et al., Laser Part. Beams 26, 241 (2008) 